\title{
From past to present (for a better future): The moderating role of cognitive mindset on spillover effects in environmental behaviors
}

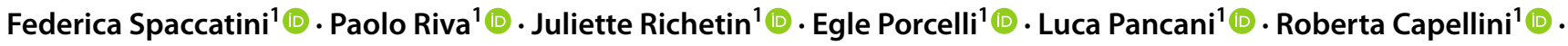 \\ Simona Sacchi ${ }^{1}$ (1)
}

Accepted: 13 February 2022

(c) The Author(s) 2022

\begin{abstract}
Research literature about the environmental spillover effect produced mixed results, revealing that an initial pro-environmental behavior (PEB) is likely to promote either other PEBs (i.e., positive spillover) or pro-environmental inactions and harming behaviors (i.e., negative spillover). Such inconsistency suggests a possible crucial role of moderating variables. In two experimental studies (N Study $1=141, \mathrm{~N}$ Study $2=124$ ), we investigated whether the recall of past environmental behavior (water-saving vs. water-wasting) affects future intention to perform PEBs (Study 1) and actual PEBs (Study 2), depending on participants' cognitive mindset (manipulated in Study 1 and measured in Study 2). Results showed that the cognitive mindset is a significant moderator of spillover effects. Compared to a holistic one, an analytical mindset is more likely to result in a greater willingness to engage in future PEBs (Study 1) and actual PEB (Study 2) when past PEB is salient. The main contributions of the studies, limitations and possible future research directions are discussed.
\end{abstract}

Keywords Spillover effect · Pro-environmental behavior · Cognitive mindset $\cdot$ Holism $\cdot$ Water consumption

The urgent need to induce people to more environmentalfriendly and sustainable behaviors has increasingly grown (e.g., Carfora et al., 2017; Thøgersen \& Noblet, 2012). A promising strategy to produce significant and long-term changes in citizens' attitudes and behaviors towards the environment lies in the spillover effect. The spillover effect is the mechanism through which an initial pro-environmental

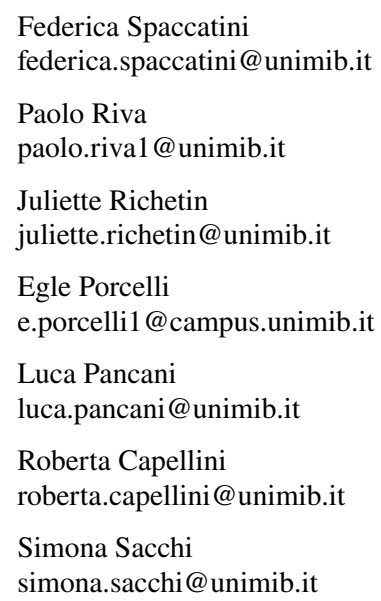

1 Department of Psychology, University of Milano-Bicocca, P.zza dell'Ateneo Nuovo 1, 20126 Milan, Italy behavior (hereafter PEB) triggers other PEBs (Nilsson et al., 2016; Verfuerth \& Gregory-Smith, 2018). However, this literature has generated mixed results, revealing that an initial PEB can promote either other PEBs or pro-environmental inactions and anti-environmental behaviors (hereafter AEBs; Nilsson et al., 2016; for a recent meta-analysis, see Maki et al., 2019). Understanding the impact and the direction of the spillover effect is fundamental to develop efficient environmental programs; thus, further research is needed to understand the circumstances under which an initial PEB triggers a virtuous circle.

In this context, the present research has been conducted to extend the research literature on spillover effects in three relevant ways. First, the available research on spillover effects has provided mixed evidence on the likelihood that a first PEB could result in further PEBs, leading to the need for an in-depth investigation of the possible moderators that can disentangle the conditions under which spillover effect could arise. We considered the cognitive mindset (analytic vs. holistic mindset) as a dispositional variable that has never been investigated in this context. Second, evidence on the spillover effect relied mostly on correlational research designs (for some exceptions, see Baca-Motes et al., 2013; Gholamzadehmir et al., 2019; Thøgersen \& Olander, 2003; 
Tiefenbeck et al., 2013). Even though correlational data provided useful insight on the phenomenon, experimental and longitudinal designs (for recent examples, see Lacasse, 2019; Sintov et al., 2019) are required to improve knowledge on the mechanism related to the spillover effect (Verfuerth \& Gregory-Smith, 2018). Therefore, in the present research, we conducted two studies adopting an experimental approach. Finally, most of the existing studies on the spillover effect have relied on self-reported behaviors or behavioral intentions (for exceptions, see Baca-Motes et al., 2013; Tiefenbeck et al., 2013), which might be affected by judgmental and social desirability biases and thus, might not reflect realistic trends. In the present research, alongside traditional self-reported behavioral intentions (Study 1), we included objective behavioral measures (Study 2).

\section{Spillover effects in the environmental domain}

In the environmental psychology literature, the spillover effect can be defined as the extent to which engaging in a PEB affects the probability of engaging in another PEB (for recent reviews, see Nilsson et al., 2016; Verfuerth \& Gregory-Smith, 2018). The second behavior can consist either in the repetition of the first behavior across time and context (temporal and contextual spillover; e.g., Littleford et al., 2014; Thøgersen \& Olander, 2003) or in a different PEB (behavioral spillover; e.g., Thøgersen \& Noblet, 2012).

\section{Positive spillover effects}

Numerous studies tested whether a first PEB would positively spill over to a second PEB, providing evidence for the virtuous escalator effect in a range of domains: from waste management behavior to sustainable shopping style (Thøgersen, 1999), from energy-saving behavior at work to energy-saving behavior at home (Littleford et al., 2014;), from "green" purchasing to a broader range of PEBs (Lanzini \& Thøgersen, 2014), and from past PEBs to concerns for climate change and support for sustainability policies (Lacasse, 2016). Thus, a positive spillover effect among different PEBs is possible, with the first PEB affecting subsequent behaviors, behavioral intentions, and policy support. However, a recent meta-analysis (Maki et al., 2019) revealed that the positive spillover effect is stronger for behavioral intentions than actual behavior. Moreover, the virtuous effect is more likely when the previous and the subsequent PEB are perceived as highly similar.

Furthermore, it is worth noting that the antecedent of the PEB is not always an actual PEB. Even making salient their pro-environmental efforts and past PEBs (e.g., Cornelissen et al., 2008; Lanzini \& Thøgersen, 2014) or asking them to commit to caring more about the environment (e.g., BacaMotes et al., 2013) triggered people to act pro-environmentally. The research revealed that pro-environmental cueing and labeling participants as "environmentalists" promoted environmental attitudes, preference for environmentalfriendly products, concerns about climate change, and support for sustainability policies (Cornelissen et al., 2008; Lacasse, 2016).

One of the most common explanations for positive spillover lies in need for consistency across behaviors, times, and contexts, using psychological theories such as the Theory of Dissonance (Festinger, 1957) and the Self-perception Theory (Bem, 1972). According to the Theory of Dissonance (Festinger, 1957), incongruences among cognitions lead people to experience internal discomfort. Thus, positive spillover might be a strategy to avoid the uneasiness of dissonance. Instead, the Self-perception Theory (Bem, 1972) posits that people infer attitudes by observing their past behaviors; thus, a first PEB might suggest a broader pro-environmental self-image, promoting the propensity to engage in other PEBs.

The positive spillover concept can also identify a vicious circle where an initial AEB might lead to other AEBs. Albeit the environmental research literature has focused on the positive spillover effect across PEBs, the literature on moral conduct has offered evidence about the progression of unethical behavior over time. For instance, Zhang et al. (2014) demonstrated that some individuals are motivated to repeat the ethical stance of a prior decision on subsequent ethical decision tasks, even when the first decision was unethical. Further, this literature provided evidence for the slippery-slope effect, according to which minor morally questionable conduct gradually allows people to engage with more severe immoral behaviors (e.g., Gino \& Bazerman, 2009; Welsh et al., 2014). Thus, in a similar vein, a prior AEB might create a de facto rule that guides and justifies future anti-environmentally behavioral choices.

\section{Negative spillover effects}

Evidence for the positive spillover has not been consistently found. Sometimes the first PEB results in a second antithetical behavior (Gholamzadehmir et al., 2019; Meijers et al., 2019; Nilsson et al., 2016; Verfuerth \& Gregory-Smith, 2018). Thus, the negative spillover effect is also possible, with a first PEB promoting either subsequent pro-environmental inaction or subsequent AEB (e.g., Mazar \& Zhong, 2010; Sütterlin \& Siegrist, 2014; Thøgersen \& Crompton, 2009; Tiefenbeck et al., 2013). For instance, Mazar and Zhong (2010) found that participants who shopped in a fictitious online store equipped with "green" (vs. conventional) products were then more likely to act immorally to gain personal profit. Negative spillover effects also emerged in a 
field study, revealing that consumers who received weekly feedback about their water consumption (vs. control group with no feedback) did reduce their water usage. However, they also increased their energy consumption (Tiefenbeck et al., 2013).

Another form of negative spillover pertains to more virtuous situations in which past AEBs motivate future PEBs. For example, highlighting the lack of past PEBs (vs. control condition) increased the subsequent willingness to request personal carbon footprint calculation (Gholamzadehmir et al., 2019).

By considering pro-environmental behaviors as a form of moral conduct, the explanation for the negative spillover generally lies in the concept of a balancing mechanism about moral self-view regulation, particularly in the moral licensing and cleansing effects. Thus, individuals who initially acted pro-environmentally might feel licensed to disengage from other environmental actions in the future (Gholamzadehmir et al., 2019; Meijers et al., 2019; Zhong et al., 2009). In contrast, individuals who initially behaved anti-environmentally might feel the need to restore their selfworth by behaving pro-environmentally (Gholamzadehmir et al., 2019; Mazar \& Zhong, 2010; Thøgersen \& Crompton, 2009).

\section{Moderators of spillover effects in the environmental domain}

Overall, research has generated mixed results, showing that past PEBs can promote or inhibit future PEBs. In light of this, researchers tried to go a step further and investigated whether and how individual differences facilitate positive spillover effects. In this regard, internal values and norms (e.g., Thøgersen \& Olander, 2003), positive attitudes towards the environment (e.g., Crompton \& Thogersen, 2009), selfefficacy (Lauren et al., 2016), and self-identity (e.g., Meijers et al., 2019; van der Werff et al., 2013) seem to play a crucial role, in line with consistency-based explanations of the phenomenon. The endorsement of norms concerning the care of the environment and more general positive attitudes towards the environment have been found to predict positive spillover (Crompton \& Thogersen, 2009; Thøgersen \& Olander, 2003).

A sizeable body of research provided consistent evidence that a positive spillover is more likely to occur and a negative spillover is less likely to happen when environmentalism is crucial to one's identity (e.g., Carfora et al., 2017; Cornelissen et al., 2008; Lacasse, 2016; van der Werff et al., 2013). Furthermore, negative spillover is more likely among people who hold weaker environmental self-identity and who, after a first PEB, feel licensed to disengage from further PEBs (Meijers et al., 2019).
However, recent research suggests that the internalization of environmentalism-related constructs does not always lead to positive spillover. Indeed, Gholamzadehmir et al. (2019) demonstrated that the frequency of past PEBs synergizes with attitudes towards the environment in determining subsequent PEB. People with stronger positive attitudes towards the environment, who frequently acted PEB in the past (vs. control condition), were less willing to request calculation of their carbon footprint. In contrast, infrequent past PEB (vs. control condition) functioned as a motivator that increased information-seeking behavior for people holding weaker positive attitudes towards the environment.

Overall, these dispositional variables focused on motivational, normative, or identity-related aspects. However, individual differences in cognitive aspects could equally influence experiences. For example, the cognitive mindset, known to play a relevant role in informing how people perceive the world, might be determinant in triggering spillover effects.

\section{The cognitive mindset}

A strand of research provided evidence that two different systems of thought exist, known as the holistic and the analytic cognitive mindset (De Oliveira \& Nisbett, 2017; Nisbett et al., 2001). A holistic mindset is characterized by attention to the context as a whole. Thus, the attention is centered on the inextricable relationship between the focal object and the context to which it belongs (Nisbett et al., 2001). An analytic mindset is, instead, characterized by a preference for details. Thus, the attention is oriented on the focal object's specific attributes, regardless of its context (Nisbett et al., 2001). Besides individual differences, even different cultures booster specific thought systems. Western cultures rely more on an analytic mindset and Eastern cultures on a holistic mindset (for reviews, see De Oliveira \& Nisbett, 2017; Markus \& Kitayama, 1991; Nisbett et al., 2001).

Cognitive mindsets can be described as a trait - stable personal-cultural-related characteristics - and state variables - characteristics that are temporarily induced by the specific situation (Förster \& Dannenberg, 2010). In this regard, it has been shown that several context-dependent variables elicit a specific cognitive mindset (e.g., the familiarity with the stimulus; one's mood; ad hoc experimental tasks; for a review, see Förster \& Dannenberg, 2010). Indeed, numerous experimental studies temporarily and successfully manipulated and induced specific cognitive mindsets throughout the adoption of tasks that function as procedural priming (e.g., Navon's task, 1977; Förster \& Dannenberg, 2010; Friedman et al., 2003; Sacchi et al., 2016).

The divergences in cognitive mindsets influence not only individual experiences due to differences in the 
construal of the self and others, and the interconnectedness of the two, but also processes of perception, cognition, and attention, motivations for action, and reasoning style (e.g., Goto et al., 2010; Kitayama et al., 2009; Nisbett \& Miyamoto, 2005; Norenzayan et al., 2002).

We hypothesized that cognitive mindsets would be determinant also in the domain of the environmental spillover effect. The literature emphasized relevant elements of mindsets that would uniquely contribute to spillover effects. For instance, attentive processes and a sense of control over the event significantly differ among mindsets (Nisbett et al., 2001). Analytical (vs. holistic) thinkers are more attentive to the focal object than its relationship with the context and perceive a greater sense of control and personal agency over the event (Cheng \& Zhang, 2017; Morris \& Peng, 1994). Thus, positive spillover would be more likely for analytical thinkers who perceived greater control over the environment through their actions. Another relevant distinctiveness among mindsets supporting our assumption is the need for consistency and avoiding contradictions. Holistic thinkers adopt a dialectical approach to tolerate and reconcile inconsistencies and contradictions and often apply the compromise principle. In contrast, analytical thinkers often rely on logical and rule-based principles, such as the law of noncontradiction, and, thus, they feel the pressure to reduce and resolve inconsistencies and contradictions (De Oliveira \& Nisbett, 2017; Nisbett et al., 2001; Peng \& Nisbett, 1999). Peng and Nisbett (1999) experimentally demonstrated the difference between dialectical and rule-based thinking in dealing with contradictions. They asked Chinese and American participants to rate the plausibility of one proposition either presented by itself or contrasted with another discordant proposition. When exposed to both propositions, trying to solve the contradiction, American participants perceived the plausible target statement as even more credible than when exposed only to it. On the contrary, Chinese participants, seeking a compromise, evaluated the more plausible proposition as less plausible than when exposed to it singularly.

Furthermore, cognitive mindset influences the construal of the self. Analytical individuals tend to hold an independent and autonomous self, whereas holistic individuals tend to hold a self-based interdependence with the context and others (for reviews, see Markus \& Kitayama, 1991; Zhu \& Han, 2008). The core elements of self-esteem and motivations to act for analytical thinkers are the consistent expression of one's capacities and essential attributes. Instead, those attributes are less determinant for holistic thinkers, who rely more on adjusting the self to situational contingencies (e.g., Markus \& Kitayama, 1991; Zhu \& Han, 2008). Thus, analytical thinkers perceive a sense of agency oriented to the self-identity affirmation.
In contrast, holistic thinkers perceive a sense of agency oriented according to a specific situation's contingencies.

The differences in dealing with inconsistency and the construal of the self also imply differences in experiencing cognitive dissonance. Analytical thinkers are more likely to experience cognitive dissonance because they avoid contradictions and are motivated to confirm their internal positive attributes (e.g., efficacy and competence; Kitayama et al., 2004). Holistic thinkers, tolerating inconsistencies, are generally less sensitive to cognitive dissonance. Kitayama et al. (2004) provided evidence of the crosscultural differences in experiencing cognitive dissonance. In particular, in a set of studies, the authors adopted the free-choice paradigm to study the cognitive dissonance after choosing between two objects (i.e., post-decisional spreading of alternatives). The results consistently revealed that American participants experienced greater cognitive dissonance than Asian participants regardless of contextual cues. Asian participants reported cognitive dissonance only in specific conditions (e.g., when social others were primed).

On these bases and also considering that the positive spillover lies in need for consistency across behaviors, times, and contexts, we reasoned that cognitive mindsets could thus modulate whether a first PEB leads to a second PEB. Furthermore, it could also be possible that the cognitive mindset and dispositional attitudes towards the environment interact in prompting positive spillover.

Throughout different operationalizations, recent research provided insights about the relevance of the cognitive mindset on environmental cognition (e.g., Davis \& Stroink, 2015; Lezak \& Thibodeau, 2016; Sacchi et al., 2016; Spaccatini et al., 2021). For instance, systems thinkers, people who hold a mindset based on interrelation among elements (i.e., for a detailed definition, see Davis \& Stroink, 2015), possessed a stronger sense of connection with nature, perceived the environmental risk as higher, expressed more support to sustainable policies, and attributed greater value to the ecosystem than non-systems thinkers (Davis \& Stroink, 2015; Lezak \& Thibodeau, 2016). Sacchi et al. (2016) hypothesized that different cognitive mindsets would be differently sensitive to environmental risk's psychological distance. Accordingly, the authors demonstrated, by both assessing and manipulating cognitive mindset, that the closer the climate change was, the more analytical (vs. holistic) thinkers expressed the sense of connection with the environment, positive attitudes towards environmentalism, and pro-environmental behavioral intentions (Sacchi et al., 2016). In brief, cognitive mindsets influence experiences, even those related to environmental issues, shaping attitudes and behavioral intentions towards the natural environment. 


\section{The Present Research}

The current research's primary aim was to investigate whether cognitive mindset interacts with recalling a past PEB (vs. AEB) in leading to the spillover effect. We asked participants to recall a past water-related (watersaving-PEB vs. water-wasting-AEB) behavior in two different experimental studies with two separate samples. In Study 1, we manipulated the cognitive mindset and assessed intentions to engage in future PEBs. In Study 2, we assessed cognitive mindset (i.e., level of holism) and objective PEBs (i.e., water and paper consumption).

Regarding a specific within-domain spillover effect, in line with the results on behaviors' similarity (Maki et al., 2019), we hypothesized that recalling a past water-related behavior would prompt a positive spillover effect (Hypothesis 1). Thus, we expected that participants who recalled a PEB (i.e., water-saving vs. water-wasting) would express more willingness to engage in future water-related PEBs (Study 1) and reduce their subsequent water consumption (Study 2). In contrast, participants who recalled an AEB (i.e., water-wasting vs. water-saving) would reduce their intention to engage in future water-related PEBs (Study 1) and increase their water consumption (Study 2). The same hypothesis was formulated regarding paper consumption, which is still a PEB but in a different domain than the initial PEB. Assuming a broader and generalizable effect of the process, recalling a past water-related behavior (PEB vs. AEB) would positively (Hypothesis 2 ) spill over to the willingness to engage in future paper-related PEBs (Study 1) and consumption of paper hand towels and paper sheet (Study 2).

Based on the evidence of the influence of the cognitive mindset on environmental cognition, attitude, and behavioral intentions, we hypothesized that the cognitive mindset would play an important role in the environmental spillover effects. We expected that the relationship between recalling past water-related behavior (i.e., water-saving vs. water-wasting) and participants' willingness to engage in future (Study 1) and observable (i.e., reducing water and paper consumptions; Study 2) PEBs would be moderated by cognitive mindsets. Specifically, cognitive dissonance functions as a motivator for positive spillover. Moreover, analytical (vs. holistic) thinkers are more sensitive to the discomfort caused by inconsistencies and cognitive dissonance. Therefore, we hypothesized that recalling past water-related PEB would foster positive spillover for people who are characterized by an analytical (vs. holistic) mindset (Hypothesis 3).

Finally, on the one hand, the positive spillover effect is more likely to occur when environmentalism is relevant to self-identity (e.g., Carfora et al., 2017; Crompton \&
Thogersen, 2009). On the other hand, we argued that positive spillover would be more likely for analytical thinkers. Thus, in an exploratory way, we tested whether people's attitudes towards the environment and their cognitive mindset interact to produce spillover effects. Alternatively, there could be a ceiling effect. People with greater environmental concerns could be less sensitive to influence exerted by their cognitive mindset because they are already attentive to act consistently in a sustainable way.

The local ethics committee has approved both experiments, and informed consent was obtained from all participants. In particular, before taking part in the study, participants have been informed of their rights to refuse to participate in the study or to withdraw consent to participate at any time during the study without reprisal. The studies were conducted following the ethical standards defined by the Declaration of Helsinki. The raw data supporting the findings of these studies are openly available in OSF at https://osf.io/9prxd/.

\section{Study 1}

\section{Design and participants}

A 2 (cognitive mindset: analytic vs. holistic) $\times 2$ (valence of the recall: water-saving - PEB vs. water-wasting - AEB) between-participants research design was adopted. Before data collection, the required sample size was computed based on a power analysis (G*Power 3.1; Faul et al., 2007). Considering the main effects of our independent variables and the two-way interactions, we needed at least 128 participants to observe a medium between-group effect size $(\mathrm{f}=0.25$, with $\alpha=0.05$ and power $=0.80$ ). The participants were recruited using a snowball procedure and the link of the online questionnaire was circulated through e-mail and social networks. A hundred and fifty-one participants (52.3\% female; $M_{\text {age }}=23.89, S D_{\text {age }}=11.93$ ) voluntarily took part in an online study. All participants were Italians, except for five individuals who had a good knowledge of the Italian language.

\section{Materials and Procedure}

Data collection was conducted online using the software Qualtrics. On the first screen, participants were informed about any relevant aspect of the study and their right to refuse to participate. They then confirmed that they understood the instructions well and expressed their consent to participate in the study. Participants were randomly assigned to one of the four experimental conditions, obtained crossing two factors, induced cognitive mindset (analytic vs. holistic) and the valence of the recalled behavior (water-saving - PEB 
vs. water-wasting - AEB). First, participants' cognitive mindset (analytical vs. holistic) was manipulated through a perception task used effectively in prior studies (e.g., Liberman \& Förster, 2009; Sacchi et al., 2016). Navon's task (1977) was used to prime either a holistic or analytical cognitive mindset between participants by instructing them to focus their attention on either the global features or the details of the presented image. Participants were presented with 14 typical Navon's stimuli (Navon, 1977). Each figure was composed of global letters formed by the configuration of local letters. They were instructed to indicate which letter was represented in the figure, choosing between two options. Whereas the correct option for participants in the analytical condition always referred to the smaller local letters, the correct option for participants in the holistic condition always referred to the bigger global letter (see Fig. 1 for an example). Thus, to select the proper answer, participants were forced to focus on the global (holistic cognitive mindset condition) or the local (analytical cognitive mindset condition) level.

Then, we manipulated the valence of water-related past behavior. Participants randomly assigned to the PEB condition were asked to write about an experience they engaged in a water-saving behavior. In contrast, those assigned to the AEB condition were asked to write about an experience in which they engaged in a water-wasting behavior.

Finally, participants were presented with a short ad-hoc questionnaire aimed to assess their willingness to reduce water and paper consumption. Three items were created to capture intentions to reduce water consumption (e.g., "If I were to brush my teeth, I would be careful about turning off the tap while doing it'). Four items were developed to assess future paper-related PEBs. Two items measured intention to recycle paper (e.g., "If I were to throw the paper sheet in the trash, I would throw it in recycling bin"). Two items measured intention to reduce paper consumption (e.g., "If I were to buy a train ticket, I would rather prefer to buy an electronic ticket"). These items were administered in a randomized order and rated on a scale ranging from $1(=n o t$ at all likely) to 7 (= very likely). After the questionnaire, participants were provided with a written debrief and were invited to contact authors for any doubt. Finally, they were thanked for their participation.

\section{Results}

\section{Preliminary analyses}

We first checked whether the content of the participants' recall was consistent with their experimental condition. Eight participants failed the recalling task, either reporting a past event inconsistent with their experimental condition or non-reporting any past event. Furthermore, we checked whether participants made errors in performing the Navon task. Two participants did not perform the Navon's task. Thus, their data was removed from the subsequent analyses, which were conducted on the remaining sample of a hundred forty-one participants ( $52.5 \%$ female; $M_{\text {age }}=23.54$, $S D_{\text {age }}=11.77$ ). We ran a sensitivity power analysis (e.g., Perugini et al., 2018) to test whether our final sample was sufficient to detect the key hypothesized effects. Based on
Fig. 1 Examples of Navon's stimuli used to manipulate the cognitive mindset (Study 1)
Which letter do you see in this image?

[analytic condition] [holistic condition]

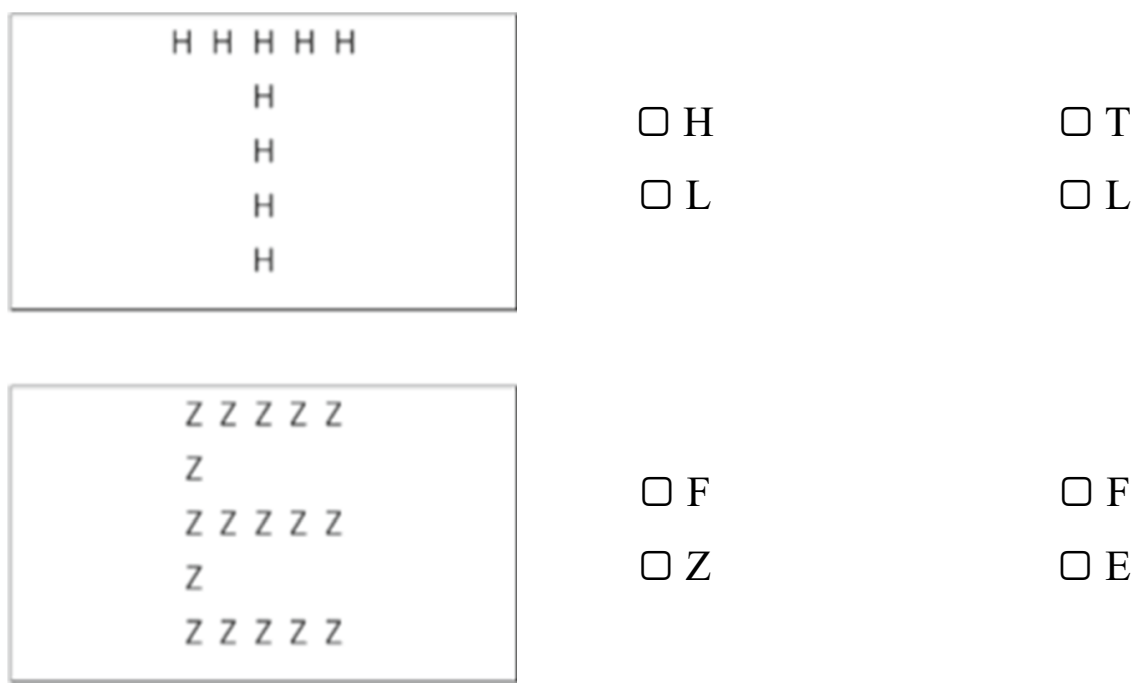


Table 1 Descriptive statistics and Correlations Among Key Variables (Study 1)

\begin{tabular}{llllll}
\hline Variables & $M$ & $S D$ & 1 & 2 & 3 \\
\hline 1. Water reduction intentions & 5.47 & 1.65 & 1 & & \\
2. Paper reduction intentions & 5.89 & 1.80 & $.21^{*}$ & 1 & \\
3. Paper recycling intentions & 6.18 & 1.32 & $.41^{* * *}$ & $.24 * *$ & 1 \\
\hline
\end{tabular}

$* p<.05, * * p<.01, * * * p<.001$

this sample of 141 participants, a sensitivity analysis with power set at 0.80 and alpha equal to 0.05 allows us determining the effect size that can be reliably detected, corresponding to $f=0$. 2376. For ease of interpretation, transforming this value as Cohen's $d$ yields 0.475 . In short, this means that our experiment had sufficient power to detect a lower than medium effect size reliably.

Reliability analyses showed a sufficient level for water reduction intentions (Cronbach's $\alpha=0.64$ ), paper recycling intentions $(r=0.25, p=0.003)$, and paper reduction intentions $(r=0.27, p=0.001)$. We computed the variables, respectively, of water reduction intentions, paper reduction intentions and paper recycling intentions as mean scores.

Table 1 reports the descriptive statistics and bivariate correlations.

\section{Effects of cognitive mindset and past behavior recall on behavioral intentions}

A 2 (cognitive mindset: analytic vs. holistic) $\times 2$ (valence of the recall: water-saving-PEB vs. water-wasting- AEB) between-participants ANOVA was conducted on each dependent variable. See Table 2 for means and standard deviations.

For the water reduction intentions, neither the cognitive mindset, $F(1,137)=0.66, p=0.418, \eta_{\mathrm{p}}{ }^{2}=0.005$, nor the valence of the recall, $F(1,137)=1.92, p=0.168, \eta_{\mathrm{p}}{ }^{2}=0.014$ emerged as significant. Thus, our first hypothesis was not confirmed. However, as hypothesized (H3) there was a significant interaction between the cognitive mindset and the valence of the recall, $F(1,137)=4.35, p=0.039, \eta_{\mathrm{p}}{ }^{2}=0.031$ (see Fig. 2).

Post-hoc comparisons showed that participants who recalled a water-saving behavior expressed greater willingness to reduce water consumption when exposed to the analytical condition compared to the holistic condition, $t(70)=2.35, p=0.01, d=0.51,95 \%$ CI $[0.08,0.95]$. In contrast, the difference between the analytic and the holistic condition was not significant for those recalling a waterwasting event, $t(67)=0.82, p=0.42, d=0.20,95 \%$ CI [-0.28, $0.67]$.

As for the ANOVA conducted on paper reduction intentions, the cognitive style, $F(1,137)=1.74, p=0.190$,
Table 2 Means and standard deviations of dependent measures as a function of cognitive style and valence of the recall (Study 1)

\begin{tabular}{llll}
\hline & \multicolumn{2}{c}{ Valence of the recall } & \\
\cline { 2 - 4 } & $\begin{array}{l}\text { Water-saving behav- } \\
\text { ior }\end{array}$ & $\begin{array}{l}\text { Water- } \\
\text { wasting } \\
\text { behavior }\end{array}$ & Combined \\
Outcome & $M(S D)$ & $M(S D)$ & $M(S D)$ \\
\hline Water reduction intentions & & \\
Analytic mindset & $6.08(1.27)$ & $5.12(1.87)$ & $5.57(1.67)$ \\
Holistic mindset & $5.28(1.61)$ & $5.47(1.69)$ & $5.37(1.64)$ \\
Combined & $5.66(1.50)$ & $5.28(1.78)$ & \\
Paper reduction intentions & & \\
Analytic mindset & $5.71(1.83)$ & $5.70(1.92)$ & $5.70(1.86)$ \\
Holistic mindset & $6.01(1.81)$ & $6.19(1.62)$ & $6.09(1.72)$ \\
Combined & $5.87(1.81)$ & $5.92(1.79)$ & \\
Paper recycling intentions & & \\
Analytic mindset & $6.16(1.11)$ & $6.41(1.18)$ & $6.29(1.14)$ \\
Holistic mindset & $6.04(1.42)$ & $6.10(1.58)$ & $6.07(1.48)$ \\
Combined & $6.10(1.27)$ & $6.27(1.37)$ & \\
\hline
\end{tabular}

$\eta_{\mathrm{p}}{ }^{2}=0.013$, the valence of recall, $F(1,137)=0.08, p=0.778$, $\eta_{\mathrm{p}}{ }^{2}=0.001$, and the two-way interaction, $F(1,137)=0.10$, $p=0.757, \eta_{\mathrm{p}}{ }^{2}=0.001$, did not show a significant effect. The ANOVA on paper recycling intentions did not reveal significant effects of the cognitive style, $F(1,137)=0.93, p=0.336$, $\eta_{\mathrm{p}}{ }^{2}=0.007$, of the valence of recall $F(1,137)=0.46$, $p=0.500, \eta_{\mathrm{p}}{ }^{2}=0.003$, and of the interaction between them, $F(1,137)=0.18, p=0.674, \eta_{\mathrm{p}}{ }^{2}=0.001$. Thus, our hypotheses on the effects of respectively the valence of the recall $(\mathrm{H} 2)$ and its interaction with cognitive mindset $(\mathrm{H} 3)$ were not confirmed.

\section{Discussion}

Study 1 provided initial evidence that the cognitive mindset moderates the relationship between past PEB and willingness to engage in future PEBs. Counter to our hypotheses, the recall's valence did not impact future behavioral intentions. However, as hypothesized, the cognitive mindset synergizes with the valence of the recall of PEBs to produce spillover effects. Specifically, participants in the analytical cognitive mindset condition after recalling a past waterrelated PEB (vs. water-wasting behavior) expressed more willingness to engage in future water-related PEBs. This effect did not emerge for participants in the holistic cognitive mindset condition.

Furthermore, the lack of interactive effect between recall and cognitive mindset on future paper-related PEBs might indicate that the effect is specific to the recalled PEB domain. Thus, we further explored this aspect in Study 2. In particular, Study 2 aimed to test further the moderating 
Fig. 2 Interaction among cognitive mindset and the valence of the recall on water reduction intentions (Study 1)

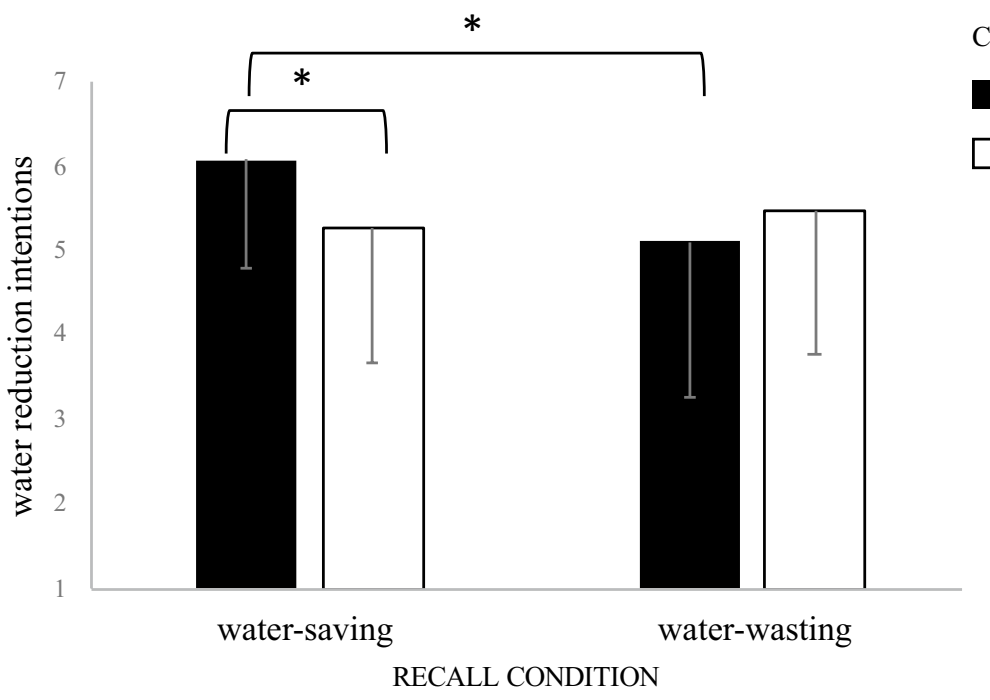

COGNITIVE MINDSET

analytic

holistic role of the cognitive mindset on the spillover effect, extending Study 1 in two ways. First, Study 1's results and most of the research literature on the spillover effect relied on self-reported behavioral intentions. In Study 2, we introduced objective behavioral measures. Second, dispositional variables such as positive attitudes towards the environment (e.g., Crompton \& Thogersen, 2009) and environmental selfidentity (e.g., Carfora et al., 2017) moderate spillover effects. Moreover, cognitive mindsets influence self-construal (Markus \& Kitayama, 1991; Zhu \& Han, 2008). We thus extended previous research literature by exploring whether pro-environmental self-identity interacts with the cognitive mindset to produce positive spillover effects.

\section{Study 2}

\section{Design and participants}

A between-participant research design was adopted, with the valence of the recall of past water-related behavior as an independent variable (PEB vs. AEB). We conducted a power analysis for sample size estimation $\left(\mathrm{G}^{*}\right.$ Power 3.1; Faul et al., 2007). With an $\alpha=0.05$ and power $=0.80$, the projected sample size needed to detect a medium effect size $\left(f^{2}=0.15\right)$ for regression with three predictors (the independent variable, the individual variable, and their interaction) was at least of 77 participants. To test our hypotheses, we advertised the study on campus throughout Sona System (C, the online participant management system that the Department uses. A hundred twenty-six Italian participants who did not participate in Study 1 (65.1\% female, $91.3 \%$ student, $M_{\text {age }}=23.13, S D_{\text {age }}=3.63$ ) were recruited and voluntarily participated in the study.

\section{Materials and Procedure}

Participants were tested individually. Each experimental session took about 30 min within the university lab. As in Study 1, before taking part in the study, participants were informed about any relevant aspect of the study and their right to refuse to participate. They then confirmed that they understood the instructions well and expressed their consent to participate in the study. According to the cover story, participants were informed that researchers were conducting a marketing study to test the features of new liquid soap and that they were interested in understanding possible consumers' attitudes towards this soap and classifying consumers according to their specific characteristics. Thus, participants were told that they would participate in three different tasks: filling in a questionnaire, evaluating and testing the new liquid soap, and drawing a logo for this product.

Before the experimental manipulation, participants completed a questionnaire to collect their basic sociodemographic information (age, gender, nationality) and measure moderator variables. Six items from the subscale Locus of Attention of the Analysis-Holism Scale (Choi et al., 2007) were used to assess our main moderating variable, that is, participants' analytic versus holistic thinking tendency (e.g., "It is more important to pay attention to the whole than its parts", Cronbach's $\alpha=0.75)$. Then, we included a measure of participants' sense of connection with the natural environment, administering ten items of the Commitment to the Environment Scale by Davis et al., (2009; e.g., "I feel very attached to the natural environment"; Cronbach's $\alpha=0.85$ ). Finally, participants' environmental activism was assessed through six items of the Pro-Environmental Behavior Scale (Dono et al., 2010; e.g., "In the political election I vote for a candidate because s/he is in favor of strong environmental protection"; Cronbach's $\alpha=0.80$ ). 
As in Study 1, participants were randomly assigned to either the PEB condition $(n=64)$ and asked to describe a past event in which they engaged in a water-saving behavior; or the AEB condition $(n=62)$ and asked to describe a past event in which they engaged in a water-wasting behavior.

To increase the cover story's credibility, participants completed a Marketing Scale assessing their consumption habits and preferences after recalling and describing their experience. Then, to justify the recalling task, participants were told that the marketing agency would analyze their writing style to capture those personality features of their consumption behavior. Participants were then presented with a liquid soap pack and asked to judge the package's likeability and the aroma of the soap as a part of the cover story. These filler items were excluded from further analyses.

The dependent variables were then measured. The first behavioral variable was water consumption. An assistant researcher brought participants, one at a time, to a sink and asked them to wash a dish with the soap they had previously evaluated. The research assistant provided each participant with the same quantity of soap. To measure our dependent variable (i.e., water consumption), the sink was equipped with a water meter (not easily visible by participants). Before and after each washing-up task, the research assistant recorded the values reported on the water meter under the sink. We computed a water consumption index by subtracting the value reported before the task from the one reported after. Higher values of the water consumption index indicated greater water consumption. This procedure and the water-related measure were previously used by Richetin et al. (2016).

The second dependent variable was paper hand towels consumption. To measure it, the research assistant made sure that the same quantity of paper hand towels was placed beside the sink for every participant. The number of paper hand towels each participant used to dry their hands were counted to form an index of paper hand towels consumption.

Following this, as a concurrent measure of participants' paper sheet consumption, participants were given 4 min to draw at least one logo for the soap they used. Each participant was provided with the same amount of paper sheets. After the drawing-log task, the paper sheet consumption index was calculated by dividing the number of drawn logos by the number of paper sheets used by each participant.

After the logo task, participants were then thanked and fully debriefed.

\section{Results}

As in Study 1, we first checked whether the content of the participants' recall was consistent with their experimental condition. Two participants failed the recalling task, either reporting a past event inconsistent with their experimental condition or not reporting any past event. Thus, their data was removed from the subsequent analyses, which were conducted on the remaining sample of a hundred twenty-four participants (66.1\% female; $\left.M_{\text {age }}=23.15, S D_{\text {age }}=3.65\right)$, who were equally distributed between the two experimental conditions (i.e., $n=62$ participants per condition). Based on this sample of 124 participants, a sensitivity analysis with power set at 0.80 and alpha equal to 0.05 allows us determining the effect size that can be reliably detected with three predictors, corresponding to $f=0.0908$. For ease of interpretation, transforming this value as Cohen's $d$ yields 0.182 . It means that our experiment had the power to detect a lower than small effect size reliably. Table 3 reports the descriptive statistics and the bivariate correlations, Table 4 reports means and standard deviations of dependent variables divided by experimental conditions.

We ran a series of moderation analyses to test our hypotheses about the effect of recalling past water-related PEB

Table 4 Means and Standard deviations of dependent variables divided by experimental condition

\begin{tabular}{llll}
\hline & $\begin{array}{l}\text { Water consump- } \\
\text { tion }\end{array}$ & $\begin{array}{l}\text { Paper hand tow- } \\
\text { els consump- } \\
\text { tion }\end{array}$ & $\begin{array}{l}\text { Paper sheet } \\
\text { consump- } \\
\text { tion }\end{array}$ \\
$M(S D)$ & $2.21(1.36)$ & $1.18(.89)$ \\
\hline $\begin{array}{l}\text { Water-wasting } \\
\text { recall }\end{array}$ & $2.80(1.56)$ & $2.02(1.01)$ & $1.08(.51)$ \\
$\begin{array}{l}\text { Water-saving } \\
\text { recall }\end{array}$ & $2.84(1.48)$ & & \\
\hline
\end{tabular}

Table 3 Descriptive statistics and Correlations Among Key Variables (Study 2)

\begin{tabular}{lllllllll}
\hline Variables & $M$ & $S D$ & 1 & 2 & 3 & 4 & 5 & 6 \\
\hline 1.Holism & 4.96 & 0.99 & 1 & & & & & \\
2. Connection with environment & 5.30 & 0.91 & .08 & 1 & & & & \\
3. Environmental activism & 2.08 & 0.98 & .10 & $.33^{* * *}$ & 1 & & & \\
4. Water consumption & 2.82 & 1.50 & -.11 & -.05 & -.10 & 1 & & \\
5. Paper hand towels consumption & 2.11 & 1.20 & .03 & -.05 & -.06 & $.20 *$ & 1 & \\
6. Paper sheet consumption & 1.10 & 0.64 & -.07 & -.14 & -.10 & .01 & .07 & 1 \\
\hline
\end{tabular}

${ }^{*} p<.05, * * p<.01, * * * p<.001$ 
(vs. AEB) on water (H1) and paper consumption (H2) and whether participants' holism moderated the relationships between the type of recall and actual behaviors towards water and paper, respectively (H3). We conducted moderation analyses using the PROCESS macro (Model 1) for SPSS with 5000 bootstrapping resamples (Hayes, 2018). For each behavior (water consumption, hand towels paper consumption, paper sheet consumption), we considered the type of recall $(0=\mathrm{AEB} ; 1=\mathrm{PEB})$ as the predictor and the $\mathrm{z}$-scores of participants' holism as the moderator.

The first model, computed on the water consumption index, did not confirm our hypothesis (H1), thus the type of recall did not significantly impact water consumption behavior, $b=-0.01, S E=0.27, t=-0.02, p=0.985,95 \% \mathrm{CI}$ $[-0.53,-0.52]$. The effect of participants' holism emerged as significant, $b=-0.48, S E=0.20, t=-2.39, p=0.019,95 \%$ CI [-0.87, -0.08]. Furthermore, as a partial support for our hypothesis (H3), the interaction between the experimental condition and participants' holism on water consumption behavior reached significance (see Fig. 3), $b=0.58$, $S E=0.27, t=2.15, p=0.034,95 \%$ CI $[0.05,1.11]$. However, the simple slope analysis only revealed a tendency.There was a positive tendency (i.e., lower water consumption in the water-saving condition than in the water-wasting condition) for participants with a lower level of holism (-1SD), $b=-0.58, S E=0.38, t=-1.53, p=0.128,95 \%$ CI [-1.34, $0.17]$. In constrast, there was an opposite negative tendency (i.e., higher water consumption in the water-saving condition than in the water-wasting condition) for those with a higher level of holism ( $+1 \mathrm{SD}$ ), $b=0.57, S E=0.38, t=1.52$, $p=0.131,95 \%$ CI $[-0.17,1.32]$. To test further our hypothesis (H3), we, then, made a comparison between the two beta-scores. This test showed that the difference between the positive tendency for participants with a lower level of holism and the opposite negative tendency for those with a higher level of holism was significant, $z=7.12, p<0.001$.

The second and the third models were computed on the paper hand towels consumption index and the paper sheet consumption index. Contrary to our expectations (H2 and $\mathrm{H} 3$ ), the main and interaction effects were non-significant, $p s>0.134$. Overall, the tested model showed that the effect of holism and the experimental condition was specific to the water-related behavior. In contrast, it did not affect other environmental-related behaviors (i.e., paper consumption).

To explore possible effects of the individual variables (i.e., connection with the environment and environmental activism), we ran additional moderation models (Model 3 ). The experimental condition $(0=\mathrm{PEB} ; 1=\mathrm{AEB})$ was entered as a predictor, $\mathrm{z}$-scores of participants' holism as a first moderator, connection with the environment and environmental activism - respectively - as a second moderator, and water consumption as the dependent variable. These models did not show any significant interactions. In particular, the three-way interactions between participants' holism and the valence of the recall with respectively connection with the environment, $b=-0.53, S E=0.30, t=-1.78, p=0.077$, 95\% CI $[-1.12,0.06]$, and environmental activism, $b=-0.15$, $S E=0.31, t=-0.47, p=0.638,95 \%$ CI $[-0.76,0.47]$ did not show significant effect on participants' water consumption.

\section{Discussion}

Study 2 provided further evidence that the cognitive mindset moderated the relationship between recalling past waterrelated PEB and subsequent water-related PEB. Even though the interaction between the valence of the recall and the cognitive mindset on subsequent water-related PEB emerged,
Fig. 3 Interaction between cognitive mindset and the valence of the recall on actual water consumption (Study 2)

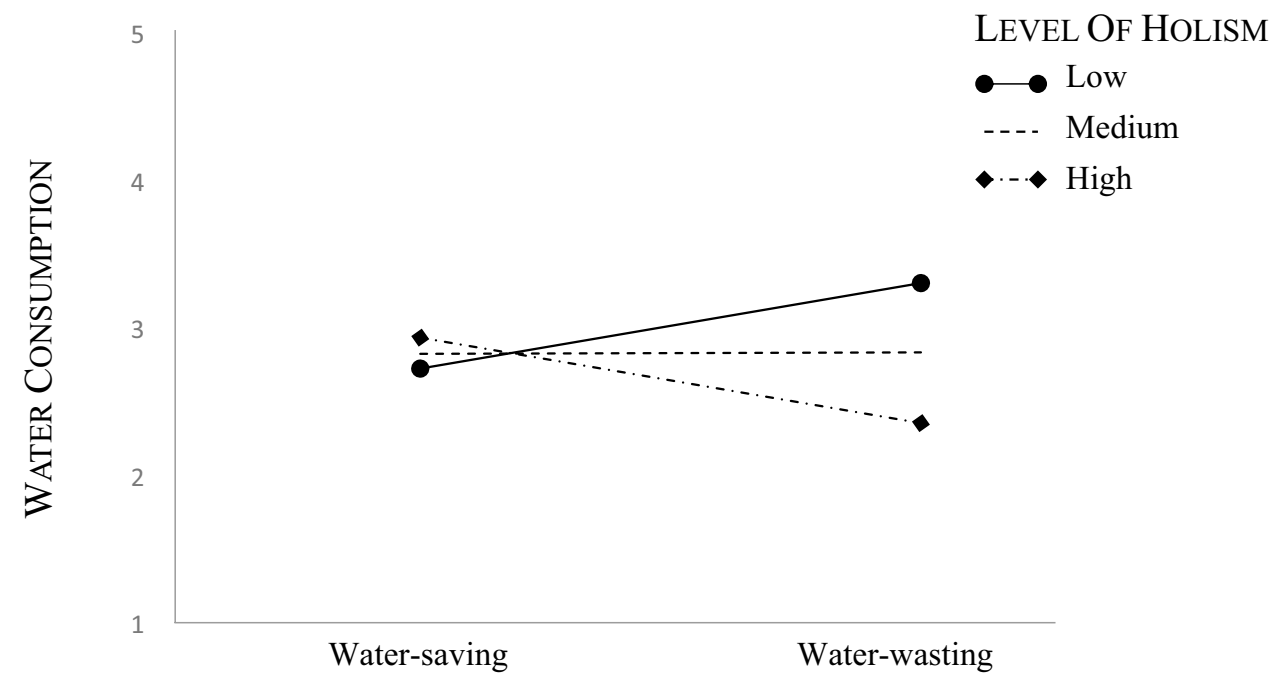

RECALL CONDITION 
none of the levels of holism reached significance. However, our analysis revealed a tendency towards a positive spillover effect for people with an analytical mindset and, conversely, a tendency towards a negative spillover effect for people with a holistic mindset. Further, in line with Study 1, the interaction between the valence of the past recalled PEB and participants' holism was significant but only within the same environmental domain (i.e., water consumption), suggesting the effect's specificity. Finally, our model revealed that the cognitive mindset, the valence of the recall, and the environmentalism variables did not produce a significant effect.

\section{General Discussion}

The urgent imperative of promoting a more sustainable and eco-friendlier lifestyle among citizens has driven academics' and policymakers' recent efforts to identify strategies for long-term attitudinal and behavioral changes. Among these strategies, existing research provided evidence of the socalled spillover effect, a mechanism through which an initial PEB triggers other environmentally sustainable behaviors (Nilsson et al., 2016; Verfuerth \& Gregory-Smith, 2018). However, the literature has offered contradictory findings of spillover effects, revealing that an initial PEB can either promote or inhibit other PEBs (Maki et al., 2019; Nilsson et al., 2016). This inconsistency revealed the need to investigate possible moderator variables that would disentangle the conditions under which spillover effects could arise.

The present research has been conducted with the specific aim of testing, for the first time, whether people's cognitive mindset would moderate spillover effects. Across two studies, we provided initial evidence that the cognitive mindset moderates the relationship between past PEB and willingness to engage in future PEBs (Study 1) and objective PEBs (Study 2).

As far as direct spillover effects are concerned, neither Study 1 nor Study 2 supported our hypotheses about positive spillover effects. Although literature provided both evidence of positive (e.g., Cornelissen et al., 2008; Lanzini \& Thøgersen, 2014) as well as negative (e.g., Gholamzadehmir et al., 2019; Nilsson et al., 2016; Tiefenbeck et al., 2013) spillover effects, there are also other research studies which met a null-effect (Maki et al., 2019). However, as expected, the spillover effect emerged when the interaction between recalling past PEB and cognitive mindset was considered.

In Study 1, we found an interaction between the recall of past PEB and cognitive mindset, with the positive spillover effect emerging for people induced to hold an analytical mindset, but not for those induced to hold a holistic one. This result corroborates the abundant literature suggesting that different cognitive mindsets result in different processes of perception and cognition (e.g., Nisbett \& Miyamoto,
2005; Nisbett et al., 2001; Norenzayan et al., 2002; Sacchi et al., 2016). A plausible explanation for the divergent effect that cognitive mindsets produce on willingness to engage in future PEBs lies in the relevant elements that qualify specific mindsets. In this case, recalling past PEBs made previous eco-friendly efforts particularly salient to analytical thinkers (Sacchi et al., 2016). Their perception of control over their environment (Cheng \& Zhang, 2017) and the need to avoid dissonance and contradictions (De Oliveira \& Nisbett, 2017) might have led them to express future behavioral intentions that were consistent with what they recalled. Our result and explanation are in line also with the rationale at the basis of positive spillover. Indeed, the need for consistency and avoiding unpleasant dissonance have been identified as core characteristics of analytical thinkers and essential motivators to positively spillover from past PEBs to future ones (e.g., Nilsson et al., 2016).

Furthermore, the spillover effect emerged only in the first PEB domain, and it did not extend to other categories of PEBs, such as future paper-related intentions. This result is consistent with evidence showing that positive spillover from a first PEB to intentions to engage in future PEBs was more likely when the past and the future PEBs are perceived as highly (vs. lowly) similar (Maki et al., 2019). Besides, the specificity of the effect is also consistent with the features of the analytical mindset. Thus, the observed spillover effect was specific because analytical thinkers are focused on water-related behaviors and, therefore, feel the need to be consistent within the domain of water-related behavior. On the contrary, recalling past PEBs did not influence future willingness to engage in PEBs for holistic thinkers. Because they are less concerned about dissonance and more attentive to the whole configuration rather than on the specific focal object, they are insensitive to the salience evoked by the recall within as well as across PEBs domains (Nisbett et al., 2001).

Study 2 sought to replicate and extend Study 1 by introducing objective PEB measures and exploring whether the cognitive mindset and environmentalism jointly lead to the spillover effect. Replicating Study 1, we found a significant interaction between the cognitive mindset and the valence of the recall on water consumption. However, a closer look at this interaction revealed a more nuanced pattern of results than Study 1. In particular, none of the holism levels were significant. Despite the lack of significance, a closer look revealed that analytical and holistic thinkers tended in the opposite directions, and the beta-comparison emerged to be significant. While recalling past water-related PEB tended to foster a positive spillover effect for analytical thinkers, it tended to facilitate a negative spillover effect for holistic thinkers.

In line with the explanation of Study 1, the results from Study 2 are consistent with the literature on both spillover 
effect and cognitive mindset. On the one hand, the interactive effect emerged only within the same category of PEBs (i.e., water-related behaviors; Maki et al., 2019). On the other hand, the positive spillover effect, which is motivated by the need for consistency (e.g., Nilsson et al., 2016), tends to characterize those individuals, i.e., analytical thinkers, who are particularly oriented to avoid contradictions. Thus, we reasoned that analytical thinkers tend to consume less water after recalling a past water-related PEB because, focused on a positive valence of the water-related issue, they behaved to avoid dissonance and maximize consistency. The determinant role of the saliency of information for analytical thinkers has been previously observed by Sacchi et al. (2016). The authors revealed that the closer climate change is perceived, and thus, salient, the more analytical thinkers drew their concerns to environmental issues expressing more pro-environmental attitudes and behavioral intentions.

When considering holistic thinkers, we found the opposite tendency. When they recalled an event in which they were careful in using water, they tended to consume more water. This tendency, albeit non-significant, seems to be in line with specific features of a holistic mindset. Holistic thinkers tended to consume more water because they are more able to integrate information from the past to the present, being less sensitive to the saliency evoked by the recall and feeling to a lesser extent the need to act consistently (De Oliveira \& Nisbett, 2017; Markus \& Kitayama, 1991; Zhu \& Han, 2008). This explanation is in line with Sacchi et al. (2016) work. Holistic thinkers were less sensitive to the saliency of the closeness of climate change because they were already more attentive to the whole configuration. This, then, determined a weaker change of concerns for the environment. Another possible explanation might be related to the licensing effect underpinning negative spillover (Gholamzadehmir et al., 2019; Meijers et al., 2019; Zhong et al., 2009). According to the licensing effect, past PEBs discourage individuals from acting other PEBs, because they perceive that they have already done their good deeds. It might be particularly true for individuals less concerned with the need for consistency, such as holistic thinkers.

Another sociopsychological framework worth to be mentioned is the Construal Level Theory (CLT; Liberman \& Trope, 2008; Trope \& Liberman, 2010). A core element of CLT is the psychological distance, which is the perceived spatial, temporal, social, and hypothetical distance of an event or an object from the self. The psychological distance shapes the level of abstraction with which individuals mentally construe a stimulus, which, in turn, influences their attitudes and behaviors (for a meta-analysis, see Soderberg et al., 2015). In particular, people mentally construe a psychologically close stimulus by focusing on feasibility (low-level construal) and psychologically distant stimulus by focusing on general purpose (high-level construal). Both cognitive mindsets and CLT have been adopted to study environmental cognition (e.g., Griffioen et al., 2019; Reczek et al., 2018; Ryoo et al., 2017; Sacchi et al., 2016; Spaccatini et al., 2021). Research showed that holistic cognitive mindset and high-level construal are related to more environmental concerns, pro-environmental intentions, and purchase intentions for eco-friendly products (Davis \& Stroink, 2015; Griffioen et al., 2019; Lezak \& Thibodeau, 2016; Reczek et al., 2018). Furthermore, prior research suggested that the construal level and the cognitive mindset are positively related, with, for instance, primed cognitive mindset influencing perceived psychological distance of events (Liberman \& Förster, 2009). In the light of this evidence, it might be possible that in our research, the cognitive mindset could have activated a different level of construal, with aneffect on the psychological distance of the recalled event. Given that we could not test this possibility, future research should disambiguate ths aspect, examining the relationship between cognitive mindset and CLT on spillover effect properly.

Although CLT and cognitive mindset are related, they refer to different constructs. Researchers investigated whether and how they jointly affect human cognition. For instance, Sacchi et al. (2016) provided evidence that the closeness of climate change elicited more positive attitudes and intentions towards the environment in analytical thinkers but not in holistic thinkers. Other research on promotion of sustainable behaviors provides evidence and inshights that the two frameworks are different (e.g., Goldsmith et al., 2016; Griffioen et al., 2019; Ryoo et al., 2017). A key difference between cognitive mindset and CLT can be inferred from the research by Ryoo and colleagues (2017). The authors demonstrated that provincial descriptive norms (i.e., how proximal people behave in a given situation; vs. general descriptive norms, i.e., how people in general behave in a given situation) encourage sustainable behaviors for low-construal level thinkers. This happens because proximal people are perceived to be psychologically closer to the self. However, while this study revealed that social others influenced low-level thinkers' intentions and behaviors, the literature on cognitive mindsets highlighted that analytical thinkers have an independent view of the self. Thus, their behavior is driven by their internal attributes rather than by others' behaviors. In light of this difference, future research should disambiguate the differences between construal levels and cognitive mindsets to shed new light on which thinking style is more effective in promoting positive spillover effect and pro-environmental attitudes.

In Study 1, the positive spillover effect from past PEB to future intention to engage in PEBs for analytical individuals emerged as fully significant; in Study 2, the more analytical individuals showed only a tendency to positively spillover from past PEB to actual PEB. Indeed, this pattern of results is not so surprising, considering that the largest 
and significant evidence for positive spillover was found for behavioral intentions to act PEB, not for actual PEBs (Maki et al., 2019).

However, while Study 1 and Study 2 provided convergent evidence about the relationship between an analytical mindset and a positive spillover effect, results about the effect of a holistic mindset on the spillover effect were less clear. Indeed, in Study 1, the spillover effect did not emerge for the holistic thinkers, whereas Study 2 highlighted that recalling past PEB tended to spillover to water consumption negatively. A possible explanation for this discrepancy might lie in the nature of adopted measures and the pressure on the consistency they evoked in participants. As noted, analytical thinkers tend to be more sensitive to the need for consistency than holistic thinkers. However, especially in Westernanalytical culture, this does not mean that holistic thinkers are not sensitive to the pressure of consistency. This might have - at least in part - influenced our results. In Study 1, we asked participants to report their willingness to engage in future PEBs. The explicit nature of this measure might have driven both analytical and holistic thinkers towards consistency. Instead, in Study 2, we relied on a behavioral measure, which was more subtle given that participants did not know that we were assessing the amount of water they consumed. Perhaps this made it possible to find a more marked effect for holistic thinkers.

The literature on the spillover effect and dispositional variables showed that previous PEBs are significant predictors of projected and actual PEBs in the future when environmentalism is perceived as an essential part of self-identity (e.g., Carfora et al., 2017; Meijers et al., 2019). We explored whether it would be universally valid across different mindsets. Our results revealed that the cognitive mindset, the valence of the recall, and the environmentalism variables were not in a significant relationship. Thus, the interaction between the cognitive mindset and the recall's valence seems to be stable and not affected by prior attitudes towards environmental issues.

Nevertheless, this research, investigating the condition under which a spillover effect could arise depending on participants' mindset, could have theoretical and practical implications. Theoretically speaking, our research could contribute to reconciling mixed results about the spillover effect. Research literature demonstrated that a first PEB could either promote or inhibit other PEBs (Nilsson et al., 2016). Our research shed new light on one of the possible reasons for the literature inconsistency. Whether past PEBs activate or not the virtuous cycle of future PEBs, at least in part, depends on the cognitive mindset, with an analytic mindset being more likely to raise a positive spillover effect.

Furthermore, in both studies, the analytical thinkers' spillover effect was restricted to water-related behavior and intentions, and it did not arise for paper-related behaviors and intentions. Even if this result aligns with the need for consistency of analytical thinkers and recent evidence about positive spillover (Maki et al., 2019), future studies should better disentangle this aspect.

In Study 1, we randomized the order of presentation of water-related and paper-related future behavioral intentions. In contrast, in Study 2, this was not possible because of the cover story and procedural constraints. Thus, future studies should ascertain whether the effect of recalling PEBs on actual PEBs might depend on the order of presentation of tasks and try to replicate our results in other environmental behavior domains such as transportations, plastic use, and more. Finally, an analytical mindset might be more likely to induce a positive spillover because analytical thinkers feel higher pressure to avoid dissonance and be consistent. However, we did not assess participants' need for consistency. Thus, future research is needed to understand better why a positive spillover is more likely for analytical thinkers than for holistic thinkers.

In line with prior studies (Sacchi et al., 2016), we provided further evidence that the cognitive mindset is not only a stable personal-cultural-related characteristic but that it would also be temporarily manipulated and induced. Our studies provided evidence that an analytical mindset, both induced and measured, is more likely to foster a positive spillover effect. Interventions and programs aimed at activating virtuous long-term cycles of PEBs should thus address their efforts to increase the impact of their communication, stimulating and inducing an adequate cognitive mindset.

Authors' contributions S. Sacchi and R. Capellini conceived the research idea. S. Sacchi, R. Capellini and E. Porcelli ran the studies. S. Sacchi conducted the data analysis and J. Richetin, P. Riva, and L. Pancani had a significant input in the data analysis. F. Spaccatini wrote the paper, the other authors read and commented on it. All authors revised and approved the final version of the paper.

Availability of data and material The raw data supporting the findings of these studies are openly available in OSF at https://osf.io/9prxd/, and materials used to collect data are available upon request to the authors.

\section{Declarations}

Ethics approval All procedures performed in studies involving human participants were conducted in accordance with the ethical standards of the institutional and/or national research committee and with the 1964 Helsinki declaration and its later amendments or comparable ethical standards. The present research protocol was approved by the Department of Psychology (University of Milano-Bicocca) Ethic Committee [Protocol number: RM-2020-253].

Consent to participate Informed consent was obtained from all individual participants included in the studies.

Conflict of interest No potential conflict of interest was reported by the authors. 
Open Access This article is licensed under a Creative Commons Attribution 4.0 International License, which permits use, sharing, adaptation, distribution and reproduction in any medium or format, as long as you give appropriate credit to the original author(s) and the source, provide a link to the Creative Commons licence, and indicate if changes were made. The images or other third party material in this article are included in the article's Creative Commons licence, unless indicated otherwise in a credit line to the material. If material is not included in the article's Creative Commons licence and your intended use is not permitted by statutory regulation or exceeds the permitted use, you will need to obtain permission directly from the copyright holder. To view a copy of this licence, visit http://creativecommons.org/licenses/by/4.0/.

\section{References}

Baca-Motes, K., Brown, A., Gneezy, A., Keenan, E., \& Nelson, L. D. (2013). Commitment and behavior change: Evidence from the field. Journal of Consumer Research, 39(5), 1070-1084. https:// doi.org/10.1086/667226

Bem, D. J. (1972). Self-perception theory. Advances in Experimental Social Psychology, 6, 1-62. https://doi.org/10.1016/S00652601(08)60024-6

Carfora, V., Caso, D., Sparks, P., \& Conner, M. (2017). Moderating effects of pro-environmental self-identity on pro-environmental intentions and behaviour: A multi-behaviour study. Journal of Environmental Psychology, 53, 92-99. https://doi.org/10.1016/j. jenvp.2017.07.001

Casey, P. J., \& Scott, K. (2006). Environmental concern and behavior in an Australian sample within an ecocentricanthropocentric framework. Australian Journal of Psychology, 58(2), 57-67. https://doi. org/10.1080/00049530600730419

Cheng, H. Y., \& Zhang, S. Q. (2017). Examining the relationship between -holistic/analytic style and classroom learning behaviors of high school students. European Journal of Psychology of Education, 32(2), 271-288. https://doi.org/10.1007/ s10212-016-0289-6

Choi, I., Koo, M., \& Choi, J. A. (2007). Individual differences in analytic versus holistic thinking. Personality and Social Psychology Bulletin, 33(5), 691-705. https://doi.org/10.1177/0146167206 298568

Cornelissen, G., Pandelaere, M., Warlop, L., \& Dewitte, S. (2008). Positive cueing: Promoting sustainable consumer behavior by cueing common environmental behaviors as environmental. International Journal of Research in Marketing, 25(1), 46-55. https://doi.org/ 10.1016/j.ijresmar.2007.06.002

Crompton, T., \& Thogersen, J. (2009). Simple and painless? The limitations of spillover in environmental campaigning. Journal of Consumer Policy, 32(2), 141-163. https://doi.org/10.1007/ s10603-009-9101-1

Davis, A. C., \& Stroink, M. L. (2015). The relationship between systems thinking and the new ecological paradigm. Systems Research and Behavioral Science, 33(4), 575-586. https://doi.org/10.1002/ sres. 2371

Davis, J. L., Green, J. D., \& Reed, A. (2009). Interdependence with the environment: Commitment, interconnectedness, and environmental behavior. Journal of Environmental Psychology, 29(2), 173-180. https://doi.org/10.1016/j.jenvp.2008.11.001

De Oliveira, S., \& Nisbett, R. E. (2017). Culture changes how we think about thinking: from "human inference" to "geography of thought". Perspectives on Psychological Science, 12(5), 782-790. https://doi.org/10.1177/1745691617702718

Dono, J., Webb, J., \& Richardson, B. (2010). The relationship between environmental activism, pro-environmental behaviour and social identity. Journal of Environmental Psychology, 30(2), 178-186. https://doi.org/10.1016/j.jenvp.2009.11.006

Faul, E., Erdfelder, E., Lang, A., \& Buchner, A. (2007). G*Power 3: A flexible statistical power analysis program for the social, behavioral, and biomedical sciences. Behavior Research Methods, 39(2), 175-191. https://doi.org/10.3758/BF03193146

Festinger, L. (1957). A theory of cognitive dissonance. Row Peterson.

Förster, J., \& Dannenberg, L. (2010). GLOMOsys: A systems account of global versus local processing. Psychological Inquiry, 21(3), 175-197. https://doi.org/10.1080/1047840X.2010.487849

Friedman, R. S., Fishbach, A., Förster, J., \& Werth, L. (2003). Attentional priming effects on creativity. Creativity Research Journal, 15, 277-286. https://doi.org/10.1080/10400419.2003.9651420

Gholamzadehmir, M., Sparks, P., \& Farsides, T. (2019). Moral licensing, moral cleansing and pro-environmental behaviour: The moderating role of pro-environmental attitudes. Journal of Environmental Psychology, 65, 101334. https://doi.org/10.1016/j.jenvp. 2019.101334

Gino, F., \& Bazerman, M. H. (2009). When misconduct goes unnoticed: The acceptability of gradual erosion in others' unethical behavior. Journal of Experimental Social Psychology, 45(4), 708-719. https://doi.org/10.1016/j.jesp.2009.03.013

Goldsmith, K., Newman, G. E., \& Dhar, R. (2016). Mental representation changes the evaluation of green product benefits. Nature Climate Change, 6(9), 847-850. https://doi.org/10.1038/nclim ate3019

Goto, S. G., Ando, Y., Huang, C., Yee, A., \& Lewis, R. S. (2010). Cultural differences in the visual processing of meaning: Detecting incongruities between background and foreground objects using the N400. Social Cognitive and Affective Neuroscience, 5(2-3), 242-253. https://doi.org/10.1093/scan/nsp038

Griffioen, A. M., Handgraaf, M. J., \& Antonides, G. (2019). Which construal level combinations generate the most effective interventions? A field experiment on energy conservation. PLOS ONE, 14(1), e0209469. https://doi.org/10.1371/journal.pone.0209469

Hayes, A. F. (2018). Introduction to mediation, moderation, and conditional process analysis: A regression-based approach (2nd ed.). The Guilford Press.

Kitayama, S., Park, H., Sevincer, A. T., Karasawa, M., \& Uskul, A. K. (2009). A cultural task analysis of implicit independence: Comparing North America, Western Europe, and East Asia. Journal of Personality and Social Psychology, 97(2), 236-255. https://doi. org/10.1037/a0015999

Kitayama, S., Snibbe, A. C., Markus, H. R., \& Suzuki, T. (2004). Is there any "free" choice? Self and dissonance in two cultures. Psychological Science, 15(8), 527-533. https://doi.org/10.1111/j. 0956-7976.2004.00714.x

Lacasse, K. (2016). Don't be satisfied, identify! Strengthening positive spillover by connecting pro-environmental behaviors to an "environmentalist" label. Journal of Environmental Psychology, 48, 149-158. https://doi.org/10.1016/j.jenvp.2016.09.006

Lacasse, K. (2019). Can't hurt, might help: Examining the spillover effects from purposefully adopting a new pro-environmental behavior. Environment and Behavior, 51(3), 259-287. https:// doi.org/10.1177/0013916517748164

Lang, K. B. (2011). The relationship between academic major and environmentalism among college students: Is it mediated by the effects of gender, political ideology and financial security? The Journal of Environmental Education, 42(4), 203-215. https://doi. org/10.1080/00958964.2010.547230

Lanzini, P., \& Thøgersen, J. (2014). Behavioural spillover in the environmental domain: An intervention study. Journal of Environmental Psychology, 40, 381-390. https://doi.org/10.1016/j.jenvp. 2014.09.006

Lauren, N., Fielding, K. S., Smith, L., \& Louis, W. R. (2016). You did, so you can and you will: Self-efficacy as a mediator of spillover 
from easy to more difficult pro-environmental behaviour. Journal of Environmental Psychology, 48, 191-199. https://doi.org/ 10.1016/j.jenvp.2016.10.004

Lezak, S. B., \& Thibodeau, P. H. (2016). Systems thinking and environmental concern. Journal of Environmental Psychology, 46, 143-153. https://doi.org/10.1016/j.jenvp.2016.04.005

Liberman, N., \& Förster, J. (2009). Distancing from experienced self: how global-versus-local perception affects estimation of psychological distance. Journal of personality and social psychology, 97(2), 203-216. https://psycnet.apa.org/doi/10.1037/a0015671

Liberman, N., \& Trope, Y. (2008). The psychology of transcending the here and now. Science, 322(5905), 1201-1205. https://doi.org/10. 1126/science. 1161958

Littleford, C., Ryley, T. J., \& Firth, S. K. (2014). Context, control and the spillover of energy use behaviours between office and home settings. Jourrnal of Environmental Psychology, 40, 157-166. https://doi.org/10.1016/j.jenvp.2014.06.002

Maki, A., Carrico, A. R., Raimi, K. T., Truelove, H. B., Araujo, B., \& Yeung, K. L. (2019). Meta-analysis of pro-environmental behaviour spillover. Nature Sustainability, 2(4), 307-315. https://doi. org/10.1038/s41893-019-0263-9

Markus, H. R., \& Kitayama, S. (1991). Culture and the self: Implications for cognition, emotion, and motivation. Psychological Review, 98(2), 224-253. https://doi.org/10.1037/0033-295X. 98.2.224

Mazar, N., \& Zhong, C. (2010). Do green products make us better people? Psychological Science, 21(4), 494-498. https://doi.org/ 10.1177/0956797610363538

Meijers, M. H. C., Noordewier, M. K., Verlegh, P. W. J., Willems, W., \& Smit, E. G. (2019). Paradoxical side effects of green advertising: How purchasing green products may instigate licensing effects for consumers with a weak environmental identity. International Journal of Advertising, 38(8), 1202-1223. https://doi. org/10.1080/02650487.2019.1607450

Morris, M. W., \& Peng, K. (1994). Culture and cause: American and Chinese attributions for social and physical events. Journal of Personality and Social Psychology, 67(6), 949-971. https://doi. org/10.1037/0022-3514.67.6.949

Navon, D. (1977). Forest before trees: The precedence of global features in visual perception. Cognitive Psychology, 9(3), 353-383. https://doi.org/10.1016/0010-0285(77)90012-3

Nilsson, A., Bergquist, M., \& Schultz, W. P. (2016). Spillover effects in environmental behaviors, across time and context: A review and research agenda. Environmental Education Research, 23(4), 573-589. https://doi.org/10.1080/13504622.2016.1250148

Nisbett, R. E., \& Miyamoto, Y. (2005). The influence of culture: Holistic versus analytic perception. Trends in Cognitive Sciences, 9(10), 467-473. https://doi.org/10.1016/j.tics.2005.08.004

Nisbett, R. E., Peng, K., Choi, I., \& Norenzayan, A. (2001). Culture and systems of thought: Holistic versus analytic cognition. Psychological Review, 108(2), 291-310. https://doi.org/10.1037/ 0033-295X.108.2.291

Norenzayan, A., Smith, E. W., Kim, B. J., \& Nisbett, R. (2002). Cultural preferences for formal versus intuitive reasoning. Cognitive Science, 26(5), 653-684. https://doi.org/10.1207/s15516709c og2605_4

Otto, S., \& Kaiser, F. G. (2014). Ecological behavior across the lifespan: Why environmentalism increases as people grow older. Journal of Environmental Psychology, 40, 331-338. https://doi.org/10. 1016/j.jenvp.2014.08.004

Peng, K., \& Nisbett, R. E. (1999). Culture, dialectics, and reasoning about contradiction. American Psychologist, 54(9), 741-754. https://doi.org/10.1037/0003-066X.54.9.741

Perugini, M., Gallucci, M., \& Costantini, G. (2018). A Practical Primer To Power Analysis for Simple Experimental Designs.
International Review of Social Psychology, 31(1), 20, 1-23. https://doi.org/10.5334/irsp.181

Reczek, R. W., Trudel, R., \& White, K. (2018). Focusing on the forest or the trees: How abstract versus concrete construal level predicts responses to eco-friendly products. Journal of Environmental Psychology, 57, 87-98. https://doi.org/10.1016/j.jenvp.2018.06.003

Richetin, J., Perugini, M., Mondini, D., \& Hurling, R. (2016). Conserving water while washing hands: The immediate and durable impacts of descriptive norms. Environment and Behavior, 48, 343-364. https://doi.org/10.1177/0013916514543683

Ryoo, Y., Hyun, N. K., \& Sung, Y. (2017). The effect of descriptive norms and construal level on consumers' sustainable behaviors. Journal of Advertising, 46(4), 536-549. https://doi.org/10.1080/ 00913367.2017.1396514

Sacchi, S., Riva, P., \& Aceto, A. (2016). Myopic about climate change: Cognitive style, psychological distance, and environmentalism. Journal of Experimental Social Psychology, 65, 68-73. https://doi.org/10.1016/j.jesp.2016.03.006

Sintov, N., Geislar, S., \& White, L. V. (2019). Cognitive accessibility as a new factor in proenvironmental spillover: Results from a field study of household food waste management. Environment and Behavior, 51(1), 50-80. https://doi.org/10.1177/00139 16517735638

Soderberg, C. K., Callahan, S. P., Kochersberger, A. O., Amit, E., \& Ledgerwood, A. (2015). The effects of psychological distance on abstraction: Two meta-analyses. Psychological Bulletin, 141(3), 525-548. https://psycnet.apa.org/doi/10.1037/bu100 00005

Spaccatini, F., Pancani, L., Richetin, J., Riva, P., \& Sacchi, S. (2021). Individual cognitive style affects flood-risk perception and mitigation intentions. Journal of Applied Social Psychology, 51(3), 208-218. https://doi.org/10.1111/jasp.12726

Sütterlin, B., \& Siegrist, M. (2014). The reliance on symbolically significant behavioral attributes when judging energy consumption behaviors. Journal of Environmental Psychology, 40, 259-272. https://doi.org/10.1016/j.jenvp.2014.07.005

Thøgersen, J. (1999). Spillover processes in the development of a sustainable consumption pattern. Journal Economic Psychology, 20(1), 53-81. https://doi.org/10.1016/S0167-4870(98)00043-9

Thøgersen, J., \& Olander, F. (2003). Spillover of environment-friendly consumer behavior. Journal of Environmental Psychology, 23(3), 225-236. https://doi.org/10.1016/S0272-4944(03)00018-5

Thøgersen, J., \& Crompton, T. (2009). Simple and painless? The limitations of spillover in environmental campaigning. Journal of Consumer Policy, 32, 141-163. https://doi.org/10.1007/ s10603-009-9101-1

Thøgersen, J., \& Noblet, C. (2012). Does green consumerism increase the acceptance of wind power? Energy Policy, 51, 854-862. https://doi.org/10.1016/j.enpol.2012.09.044

Tiefenbeck, V., Staake, T., Roth, K., \& Sachs, O. (2013). For better or for worse? Empirical evidence of moral licensing in a behavioral energy conservation campaign. Energy Policy, 57, 160-171. https://doi.org/10.1016/j.enpol.2013.01.021

Thornton, L., Batterham, P. J., Fassnacht, D. B., Kay-Lambkin, F., Calear, A. L., \& Hunt, S. (2016). Recruiting for health, medical or psychosocial research using Facebook: Systematic review. Internet Interventions, 4, 72-81. https://doi.org/10.1016/j.invent. 2016.02.001

Trope, Y., \& Liberman, N. (2010). Construal-level theory of psychological distance. Psychological Review, 117(2), 440-463. https:// psycnet.apa.org/doi/10.1037/a0020319

Van der Werff, E., Steng, L., \& Keizer, K. (2013). It is a moral issue: The relationship between environmental self-identity, obligation-based intrinsic motivation and pro-environmental behavior. Global Environmental Change, 23(5), 1258-1265. https://doi.org/ 10.1016/j.gloenvcha.2013.07.018 
Verfuerth, C., \& Gregory-Smith, D. (2018). Spillover of pro-environmental behaviour. In V. K. Wells, D. Gregory-Smith, \& D. Manika (Eds.), Handbook of employee pro-environmental behaviour (pp. 455-484). Edward Elgar Publishing.

Welsh, D. T., Ordóñez, L. D., Snyder, D. G., \& Christian, M. S. (2014). The slippery slope: How small ethical transgressions pave the way for larger future transgressions. Journal of Applied Psychology, 100(1), 114-127. https://doi.org/10.1037/a0036950

Wray-Lake, L., Flanagan, C. A., \& Osgood, D. W. (2010). Examining trends in adolescent environmental attitudes, beliefs, and behaviours across three decades. Environment and Behavior, 42, 61-85. https://doi.org/10.1177/0013916509335163

Zhang, S., Cornwell, J. F., \& Higgins, E. T. (2014). Repeating the past: Prevention focus motivates repetition, even for unethical decisions. Psychological Science, 25(1), 179-187. https://doi.org/10. $1177 / 0956797613502363$
Zhong, C.-B., Liljenquist, K. A., \& Cain, D. M. (2009). Moral selfregulation: Licensing \& compensation. In D. De Cremer (Ed.), Psychological perspectives on ethical behavior and decision making (pp. 75-89). Information Age.

Zhu, Y., \& Han, S. (2008). Cultural differences in the self: From philosophy to psychology and neuroscience. Social and Personality Psychology Compass, 2(5), 1799-1811. https://doi.org/10.1111/j. 1751-9004.2008.00133.x

Publisher's note Springer Nature remains neutral with regard to jurisdictional claims in published maps and institutional affiliations. 